\title{
tic\&société
}

Vol. 14, $\mathbf{N}^{\circ}$ 1-2 | 1er semestre 2020 - 2ème semestre 2020

Mutations numériques de la musique : des

contradictions à analyser

\section{L'autoformation, nouveau paradigme de développement de carrière dans le contexte numérique? Regards sur le milieu musical montréalais}

Being self taught, a new digital career development paradigm? Exploring the Montreal music scene

La autoformación; ¿nuevo paradigma de desarrollo de la carrera profesional en el contexto digital?. Una mirada al medio musical montrealés

Sylvain Martet, Martin LUSSIER et Anouk BÉLANGER

\section{OpenEdition}

\section{Édition électronique}

URL : http://journals.openedition.org/ticetsociete/4907

DOI : 10.4000/ticetsociete.4907

\section{Éditeur}

Association ARTIC

Édition imprimée

Pagination : 157-184

Référence électronique

Sylvain Martet, Martin LUSSIER et Anouk BÉLANGER, «L'autoformation, nouveau paradigme de développement de carrière dans le contexte numérique ? Regards sur le milieu musical montréalais », tic\&société [En ligne], Vol. 14, № 1-2 I 1er semestre 2020 - 2ème semestre 2020, mis en ligne le 11 novembre 2020, consulté le 25 février 2021. URL : http://journals.openedition.org/ticetsociete/4907 ; DOI : https://doi.org/10.4000/ticetsociete.4907 
tic\&société - 14(1-2), 2020

\title{
L'autoformation, nouveau paradigme de développement de carrière dans le contexte numérique ? Regards sur le milieu musical montréalais
}

\begin{abstract}
Sylvain MARTET
Sylvain Martet est chercheur postdoctoral à la Faculté de communication de l'Université du Québec à Montréal (UQAM), chargé de cours au Département de communication sociale et publique de I'UQAM et professionnel de recherche à Artenso, Centre collégial de transfert en pratiques sociales novatrices affilié au cégep de Saint-Laurent à Montréal. Ses recherches portent sur la circulation de la musique, le travail dans les milieux des arts et de la culture et la médiation culturelle. II est membre du laboratoire Culture populaire, connaissance et critique (CPCC) et de l'International Association for the Study of Popular Music - Canada. martet.sylvain@uqam.ca
\end{abstract}

\section{Martin LUSSIER}

Martin Lussier est professeur au Département de communication sociale et publique de l'UQAM. II s'intéresse aux musiques populaires et aux pratiques des organismes qui coordonnent les artistes, interviennent auprès d'eux et permettent d'articuler des politiques, des industries, des pratiques artistiques, des publics ainsi que des travailleurs culturels. Membre du laboratoire Culture populaire, connaissance et critique (CPCC) ainsi que du Centre de recherche interuniversitaire sur la communication, l'information et la société (CRICIS), il a publié en 2011 un livre dédié aux musiques émergentes à Montréal intitulé Les musiques émergentes. Le devenir-ensemble (Éditions NotaBene) et a codirigé, en 2019, Essor de la culture au XXlème siècle (PUM, avec M. Paquin, M. Lemonchois et J.-M. Lafortune). II codirige avec Anouk Bélanger l'Atelier de chronotopies urbaines, un dispositif de recherche et de collaboration sur les cultures populaires. lussier.martin@uqam.ca 
L'autoformation, nouveau paradigme de développement de carrière dans le contexte numérique? Regards sur le milieu musical montréalais

\section{Anouk BÉLANGER}

Anouk Bélanger est professeure au Département de communication sociale et publique de I'UQAM. Son enseignement et ses recherches portent sur les cultures populaires, les cultures urbaines et médiatiques. Elle est membre du Centre de recherche interuniversitaire sur la communication, l'information et la société (CRICIS) et dirige l'axe " Praxis » de l'Observatoire de la médiation culturelle (OMEC). Elle complète la codirection d'un numéro thématique double de la revue GLOBE portant sur les mutations de la télévision et la culture québécoise (automne 2020). Avec Martin Lussier, elle dirige l'Atelier de chronotopies urbaines.

belanger.anouk@uqam.ca 


\title{
L'autoformation, nouveau paradigme de développement de carrière dans le contexte numérique ? Regards sur le milieu musical montréalais
}

\begin{abstract}
Résumé : Cet article propose de porter un regard sur les modes de formation des travailleurs.euses du milieu musical montréalais dans l'environnement numérique. Face aux changements que celui-ci entraîne pour les métiers de la musique, les professionnels.les ont tendance à se tourner davantage vers l'autoformation plutôt que vers les offres institutionnelles pourtant bien présentes au Québec. En plus de formations ponctuelles tout au long de la carrière, les musiciens.nes et les travailleurs.euses de l'industrie musicale favorisent le développement de savoirs par l'expérience, le recours aux communautés de pratiques et la mobilisation de ressources en ligne.
\end{abstract}

Mots clés: musique, formation, numérique, autodidacte, communauté de pratique.

\section{Being self taught, a new digital career development paradigm? Exploring the Montreal music scene}

\begin{abstract}
This article examines the incidence of the digital context on the training of those working in the Montreal music scene. As digital technologies have resulted in major changes in the music industry, our research shows that professionals tend to prefer to train themselves rather than attending some of the many courses offered by Quebec institutions. In addition to periodic training throughout their careers, both musicians and technicians in the music industry tend to prefer experiential learning, drawing on communities of practice and the use of online resources.
\end{abstract}


L'autoformation, nouveau paradigme de développement de carrière dans le contexte numérique? Regards sur le milieu musical montréalais

Keywords: music, training, digital, self-education, community of practice.

\title{
La autoformación; ¿nuevo paradigma de desarrollo de la carrera profesional en el contexto digital?. Una mirada al medio musical montrealés
}

\begin{abstract}
Resumen: Este artículo analiza los recorridos de formación de los trabajadores de la industria musical de Montreal en el entorno digital. Frente a los cambios que esto implica para las profesiones musicales, se muestra que los profesionales tienden a centrarse más en la autoformación que en las ofertas institucionales, que en Québec son muy abundantes. Además de formaciones puntuales a lo largo de su carrera, músicos y otros trabajadores de la industria de la música fomentan el desarrollo del conocimiento a través de la experiencia a la vez que recurren a las comunidades de prácticas y a los cursos en línea.
\end{abstract}

Palabras claves: música, formación, digital, autodidacta, comunidad de prácticas. 


\section{Sylvain MARTET, Martin LUSSIER, Anouk BÉLANGER}

En février 2017 s'amorçaient sous le thème « Numérique et arts de la scène » les rencontres annuelles du RIDEAU (Réseau indépendant des diffuseurs d'événements artistiques unis), le plus grand réseau de diffuseurs professionnels de spectacles au Québec. À l'ouverture de l'événement, la directrice générale lançait "L'avenir est déjà derrière nous » (Brouillé, dans Papineau, 2017, p. B8), exprimant ainsi le sentiment de vitesse par laquelle le numérique s'est immiscé au cœur des pratiques de ses membres. Les différents ateliers tenus dans le cadre de ces rencontres faisaient écho à cette inquiétude : une adaptation urgente s'imposait. Ce sentiment n'est pas nouveau, il a été largement partagé au sein des divers secteurs des industries musicales et relayé dans la presse : le disque (Bourgault-Côté, 2009), l'enregistrement et les studios (Brunet, 2015) ou l'enseignement (Daoust-Boisvert, 2011), pour ne nommer que ceux-là, ont tous fait l'objet d'une couverture journalistique les liant à la « crise » occasionnée par le numérique (Grenier, 2011). Pour les membres présents lors des rencontres de 2017 du RIDEAU, l'un des enjeux principaux était la formation et la compétence des travailleurs.euses culturels. Trouver des personnes avec les connaissances adéquates et favoriser le développement de compétences numériques semblait un défi important, alors que certains.es intervenants.es en ont « profité pour plaider en faveur de la formation en continu des travailleurs culturels » (Papineau, 2017, p. B8). Cette idée que la formation est l'une des pistes à favoriser afin de pallier l'incertitude liée aux transformations récentes ressort également fortement d'un ensemble de rapports publiés au cours des dernières années concernant l'état des industries culturelles au Québec (CRHSC, 2011 ; Culture Montréal, 2016 ; Québec, 2014).

Cet article porte un regard sur les cursus et les processus suivis sciemment par les travailleurs.euses du milieu musical montréalais dans une perspective de développement de leurs compétences professionnelles dans l'environnement numérique. Pour comprendre ces modes de formation, il propose de partir d'une série d'entretiens longs réalisés en 2018 auprès de professionnels.les de la musique à Montréal, tant du côté des 
L'autoformation, nouveau paradigme de développement de carrière dans le contexte numérique? Regards sur le milieu musical montréalais

musiciens.nes que des métiers de soutien ${ }^{1}$. Par travailleurs.euses, nous entendons ici les personnes professionnelles exerçant tant des métiers créatifs (musiciens.nes, auteurs-compositeurs-interprètes, etc.) que des métiers de soutien (producteurs, gérants, éditeurs, etc.). Les entretiens semi-directifs visaient à comprendre les effets du numérique sur les métiers de la musique et les pratiques quotidiennes des travailleurs.euses, en offrant un point de vue privilégié sur les transformations qui s'opèrent dans le milieu et qui accompagnent le déploiement du numérique ${ }^{2}$. Quels types de formations sont utilisés ou valorisés par les personnes rencontrées? Quelles conceptions de la formation sont-elles partagées? Quels sont les enjeux de formation dans un environnement numérique en changement?

\section{1. Éléments de contexte : l'offre de formation aux métiers de la musique au Québec}

De manière générale, et depuis la fin des années 1970, les formations spécialisées dans les aspects techniques et administratifs de la musique se multiplient en Amérique du Nord. Thomas Porcello (2004) remarque qu'entre 1980 et le début des années 2000, leur nombre a quadruplé, atteignant 108 formations techniques délivrant un diplôme collégial ou universitaire en 2001. Cela n'est pas sans lien avec l'arrivée massive de technologies numériques (telles que le MIDI ou l'informatique) dans les studios d'enregistrement et les salles de concert au cours de la même période :

Pratiquement tous ces programmes sont fortement axés sur la formation des étudiants à l'intégration des équipements audio numériques et des ordinateurs dans leur pratique professionnelle. Cet élément est apparemment central dans la prolifération des programmes ces vingt dernières années. Ils ont, dans un sens, été

\footnotetext{
1 Ces entrevues ont été menées dans le cadre du projet de recherche Nouveaux métiers et acteurs de la culture au Québec (CRSH, Développement Savoir, 20172019). Nous tenons à remercier Andrew Duquette-Boyle pour sa participation au terrain de recherche.

${ }^{2}$ Afin de respecter la confidentialité des personnes interviewées, nous avons assigné un code de deux lettres majuscules au hasard à chacun.e des participants.es lors de la transcription des entrevues afin de retirer tout identificateur direct ou indirect. Nous nous référons donc aux participants.es par ces codes dans cet article.
} 


\section{Sylvain MARTET, Martin LUSSIER, Anouk BÉLANGER}

largement réactifs aux plus gros changements technologiques de la production musicale depuis les années $1980^{3}$ (Porcello, 2004, p. 736).

Plus spécifiquement, à Montréal s'ouvrait en 1979 l'une des premières écoles privées de formation technique en production musicale: I'Institut Trebas. Celle-ci, tout comme les autres structures de formations qui verront le jour au cours des années suivantes sur le territoire québécois, mobilise dès les premiers mois et de manière importante le numérique pour justifier sa pertinence. Suivant ce courant, en 1990, une autre école annonçait « forme[r] des gens qui pourront gagner leur vie dans les métiers liés à la conception sonore assistée par ordinateur » (Barcelo, 1990, p. 16). Ce discours mobilisant le numérique semble généralisé à l'ensemble des formations en production musicale et audiovisuelle au Québec (Charette, 2000), dont plusieurs se décrivent comme des avenues de choix dans l'accession à des métiers en changement.

Le développement de formations en production musicale s'insère dans un mouvement plus large de professionnalisation du champ artistique au Québec qui s'est déployé au cours du XXème siècle, notamment par les écoles et les universités. Audelà de la formation artistique des créateurs, qui s'amorce au Québec dans la première moitié du XXème siècle, la professionnalisation relèverait plutôt de l'émergence "d'un milieu professionnalisé, formé d'intermédiaires " autres que les artistes, permettant de structurer la pratique :

la professionnalisation du champ artistique concerne moins les occupations artistiques au sens strict, même élargies aux nouveaux emplois créatifs du secteur des médias et de la fonction publique, que celles du plus vaste champ organisationnel au sein duquel évoluent les artistes (Bellavance et Laplante, 2002, p. 318).

Depuis la fin des années 1990, l'offre de formation continue provenant d'associations diverses s'ajoute au paysage. La SOPREF (Société pour la promotion de la relève musicale de

\footnotetext{
3 Traduction libre de : « Shared by virtually all these programs - and apparently central to their proliferation in this 20-year time period - is an intense focus on training students to integrate digital audio equipment and computers into their professional practice. These programs have, in one sense, been largely reactive to the major technological changes in music production since the early 1980s ».
} 
L'autoformation, nouveau paradigme de développement de carrière dans le contexte numérique? Regards sur le milieu musical montréalais

l'espace francophone) $)^{4}$, par exemple, qui avait une mission de professionnalisation, a tenté de répondre à un besoin de formation à partir de 1998, non pas sur les dimensions musicales et artistiques, mais bien sur des aspects organisationnels, légaux et promotionnels (les contrats, le marketing, les nouvelles technologies ou la gestion de carrière) par la diffusion de contenus pédagogiques et l'organisation d'autres activités pour le milieu (Lussier, 2017). Elle voulait pallier un manque de " "compréhension des enjeux et des pratiques du milieu" industriel, légal et institutionnel au sein duquel s'insèrent ses membres » (Lussier, 2017, p. 171). Les actions de la SOPREF représentent le mouvement plus large de professionnalisation se déployant depuis le XXème siècle et s'intensifiant depuis les années 1990 au Québec et incluant divers intermédiaires nécessaires à l'organisation de la pratique artistique.

Tel que le souligne Porcello (1990), non seulement ces formations mettent l'accent sur le numérique et les compétences qu'il nécessite, mais elles permettent aussi de se familiariser avec les manières de faire dans le milieu musical, l'ensemble des aptitudes sociales permettant de développer des savoirs tacites et le langage technique permettant d'intégrer la profession. Dans les termes de la sociologie du travail artistique, il s'agit de miser sur toutes les tâches connexes à la réalisation d'un projet musical ou artistique (Bureau et Shapiro, 2009), de la conception à la diffusion, en passant par l'écriture, l'enregistrement ou la production. Dans cette même optique, l'Association québécoise de l'industrie du disque, du spectacle et de vidéo (ADISQ), créée en 1978, offre un programme de formation continue et de conférences depuis 2002. À titre d'exemple, la programmation 2018-2019 compte trois microprogrammes portant directement sur le contexte numérique (les droits d'auteur dans l'industrie musicale et dans l'univers numérique musical, les stratégies web et les métadonnées), qui s'ajoutent à diverses formations sur les métiers de l'industrie et à une formule petit-déjeuner conférence visant une compréhension de l'industrie et de ses enjeux (" La cartographie de l'industrie québécoise et canadienne de la musique ", "Le portrait de la consommation de la musique au Québec et à travers le monde »,

\footnotetext{
${ }^{4}$ Du Forum des musiques amplifiées (1997-1998) découle la création de la SOPREF, qui a existé jusqu'en 2009, dont la mission sera de professionnaliser les musiques émergentes.
} 


\section{Sylvain MARTET, Martin LUSSIER, Anouk BÉLANGER}

"La radiodiffusion : comprendre les quotas et les contributions au développement du contenu canadien »).

Ce bref panorama nous permet de constater que l'offre éducative et de formations est riche au Québec et se déploie tout en se diversifiant pour accompagner l'arrivée du numérique. Les formations en musique sont en grande partie professionnalisantes et visent l'acquisition d'un ensemble d'aptitudes sociales. Pourquoi alors l'impression de sousformation continue-t-elle d'alimenter le discours de crise lié au numérique dans le milieu ? Est-ce à dire que ces formations ne parviennent pas à s'adapter à un contexte trop versatile ? S'ils ne trouvent pas les ressources dans leur formation initiale, où puisent donc les travailleurs.euses de la musique pour le développement de leurs connaissances et de leurs carrières ?

Pour aborder cette problématique, nous avons cherché à comprendre le rapport des travailleurs.euses de la musique au paysage de formation québécois dans le cadre d'une recherche qui abordait l'ensemble des effets du numérique sur les carrières en musique. Notre corpus d'entrevues ne vise pas l'exhaustivité, mais plutôt à mettre en lumière la diversité des parcours. Le recrutement a été réalisé en mettant à contribution les différents réseaux relationnels des chercheurs.es, puis par bouche-àoreille au sein des différentes scènes musicales concernées. Sur les dix entrevues longues que nous avons menées avec des professionnels.les de l'industrie aux parcours jalonnés d'une production intensive, trois personnes possèdent un diplôme universitaire (deux en musique et un en production culturelle), deux ont suivi une formation collégiale (un cégep et un collégial privé) et les cinq autres sont autodidactes, sans cursus initial dans la musique. Les parcours en autodidacte, et plus largement les carrières en constante évolution sur la plan de l'acquisition de compétences et de connaissances, sont caractéristiques de notre corpus. Pour les comprendre, il est nécessaire de les lier aux effets du numérique et aux tactiques d'autoproduction déployées par les artistes. 
L'autoformation, nouveau paradigme de développement de carrière dans le contexte numérique? Regards sur le milieu musical montréalais

\section{Les incidences du numérique et l'entrepreneuriat culturel}

Avec le numérique, on observe une démocratisation de la production culturelle par l'intermédiaire de nouveaux outils (Le Guern, 2012), une multiplication des formes et des pratiques artistiques (Prior, 2012) ainsi qu'une circulation renouvelée des produits (Bastard et al., 2012 ; Beuscart, 2008). Dans ce contexte, une nouvelle génération d'artistes valorise de plus en plus l'autoproduction en musique (Bacache-Beauvallet, Bourreau et Moreau, 2009 ; Watson, 2016).

«Pour enregistrer, il nous faut une carte de son, un micro, un laptop, et on aime jouer aussi avec les styles beaucoup, donc j'ai plein de plug-ins qui sont dans mon ordi. » (JT)

Les compétences nécessaires à l'utilisation de ces outils ne sont pas toujours développées dans le cadre de formations scolaires aux métiers de la musique. L'utilité des formations offertes par les institutions est même mise en doute par les personnes rencontrées en considérant la vitesse avec laquelle les technologies numériques en création se transforment ou deviennent obsolètes :

«Pour tout ce qui est technique, [je n'ai] pas tant [suivi de formation]. Parce que ça évolue tellement vite que pour être up to date... [...] J'ai de la misère à croire qu'on peut institutionnaliser ces domaines-là. » (EV).

Dans le même sens, une musicienne décrit la formation collégiale et les technologies qui sont enseignées et utilisées en classe comme étant obsolètes :

"Au cégep on apprenait, mais c'était désuet. On apprenait Reason [un logiciel de musique assisté par ordinateur] et DP [Digital Performer, séquenceur multipiste/MIDI décrit comme un DAW] qui sont des vieux trucs que personne n'utilise [...], pour nous c'était plus pour faire de la musique par image, on avait eu un projet là-dessus, et on avait appris la base de Ableton [un séquenceur multipiste] un petit peu. " (JT)

Dans tous les cas, la formation initiale postsecondaire en école n'apparaît pas comme un lieu pertinent ou suffisant pour se tenir à jour des transformations technologiques. La vitesse à laquelle les changements apparaissent appelle plutôt une 


\section{Sylvain MARTET, Martin LUSSIER, Anouk BÉLANGER}

formation en continu, où les apprentissages et les activités se font en fonction des besoins des projets.

Cet environnement en mutation occasionne des tensions et crée des zones d'ambiguïtés par rapport aux fonctions et aux frontières traditionnellement associées aux métiers de la culture (Hracs, Seman et Virani, 2016). La production scientifique a montré comment les acteurs et les industries explorent des stratégies d'adaptation et des manières d'intégrer le numérique dans leurs modèles d'affaires. Dans cette même foulée, les circuits de création, de diffusion et de consommation sont quelque peu bousculés par l'arrivée de nouvelles pratiques, de nouveaux acteurs, ou par la nécessité pour ceux déjà en place de redéfinir leurs activités (Chircu et Kaufmann, 1999). Ce contexte de travail concurrentiel doublé du contexte numérique, qui donne accès à une plus grande autonomie vis-à-vis du processus de formation, de production, de distribution et de diffusion, facilite des logiques entrepreneuriales (Bureau, 2006) : aujourd'hui plus que jamais, les travailleurs.euses culturels.les sont des centres de relations, de gestion de contrats divers et doivent parfois devenir leur propre entreprise (Greffe, 2012 ; Morris, 2013).

\section{Le défi posé par la complexité des parcours et des métiers du domaine musical pour les formations}

Les travailleurs.euses de la musique, et plus largement les travailleurs.euses des milieux culturels, ont une propension à mener plusieurs projets en même temps (McRobbie, 2002), ce qui porte à la pluriactivité - c'est-à-dire l'exercice de plusieurs métiers au sein d'un même champ artistique - ainsi qu'à la polyactivité - le fait d'occuper plusieurs emplois à la fois, parfois à l'extérieur des terrains de la culture (Bureau et Shapiro, 2009). Dès lors, l'idée de métier n'est pas unidimensionnelle et, de fait, est complexe à expliquer :

«Mon métier c'est auteur-compositeur. Pour d'autres, je fais de la composition pour des musiques de films ou de série, auteur-compositeur-interprète dans mon cas, puis marketer, je fais des affaires, du online marketing, je fais du branding parce que c'est obligatoire d'avoir une image 
L'autoformation, nouveau paradigme de développement de carrière dans le contexte numérique? Regards sur le milieu musical montréalais

publique sur les réseaux sociaux, et donc je fais des réseaux sociaux, à mon grand désarroi... » (AZ)

À cette difficulté de cerner le métier, d'en faire un objet bien délimité pour lequel des formations menant à un diplôme peuvent être proposées, s'ajoute l'impression que « tu peux pas aller à l'école pour apprendre ça » (FT). En effet, pour plusieurs de nos répondants.es, le métier lui-même est fondé sur un ensemble de connaissances qui ne sont pas transférables, par exemple l'évaluation de la valeur d'une performance musicale, la gestion des rapports avec les fans sur les réseaux sociaux ou encore les tactiques de monétisation des produits musicaux. Le doute face à l'offre de formation dans des écoles est ainsi fondé sur l'impression, pour reprendre l'expression utilisée par une personne rencontrée, que "ça ne s'enseigne pas ce que je fais " $(K R)$. L'impossibilité d'enseigner le métier ou les tâches quotidiennes semble en partie tributaire de la difficulté qu'ont plusieurs travailleurs.euses à exprimer ou à décrire leur métier. Ainsi, malgré la participation de ces formations à la professionnalisation du milieu musical par l'enseignement de savoirs tacites, des formes importantes de connaissances demeurent à l'extérieur du giron des écoles.

Le travail quotidien d'appui à la pratique artistique fait l'objet d'un nombre croissant de recherches, s'attardant à documenter les tâches qui rendent possible la pratique musicale (communication, marketing, financement, coordination, etc.) (Baker et Hesmondhalgh, 2011; Menger, 2002) : "Le travail administratif, au sens large, est une partie intégrante du "métier d'artiste" et non une activité accessoire ou optionnelle " (Sinigaglia-Amadio et Sinigaglia, 2017, p. 76). Les transformations récentes des industries musicales en contexte numérique, les métiers émergents et l'importance grandissante de certaines dimensions de la carrière musicale qui en découlent induisent des tâches et des compétences nouvelles. Celles-ci varient considérablement selon les projets, les types de carrière et l'entourage professionnel. Ceci dit, la formation aux savoirs tacites du milieu musical, ou, en d'autres termes, apprendre « en faisant » le métier et être en position d'évolution constante du point de vue des compétences, s'impose aujourd'hui. Par exemple, une personne rencontrée se décrivant comme superviseur musical souligne : 


\section{Sylvain MARTET, Martin LUSSIER, Anouk BÉLANGER}

« C'est un métier encore un peu embryonnaire, À l'époque où j'ai commencé, on apprenait beaucoup en faisant. Aujourd'hui encore j'apprends des trucs. [...] C'est plus un apprentissage du quotidien. C'est très empirique en ce qui me concerne. Je n'ai pas de formation. " (LS)

Avec la transformation du travail musical en contexte numérique, l'expérience est non seulement celle d'un métier et de ses savoirs implicites, mais aussi celle de la constitution du métier en tant que tel. C'est à ce titre un mode essentiel de l'autoformation.

\section{L'expérience et la formation en autodidacte}

"La formation c'était la route. C'était d'être en tournée, on peut comprendre c'est quoi être un artiste sur la route. » (HR)

L'expérience est souvent décrite comme une évidence, un cumul de vécu ou une pratique qui s'est incorporée. Elle demeure un objet d'attention chez les chercheurs qui s'intéressent à ce qu'elle autorise tout comme à ce qui la constitue discursivement, notamment :

L'expérience implique un domaine solide, concret et certain, en opposition à l'abstraction vague de la philosophie et de la théorie sociale. Elle confère souvent de l'autorité lorsqu'elle est associée à l'expérience directe de la vie, par opposition à "l'apprentissage dans un livre », et sert souvent de garantie de vérité de bon sens et de témoin: "Je sais parce que j'étais là » ${ }^{5}$. (Bérubé, 2005, p. 122.)

L'expérience de scène apparaît comme un fondement majeur de la formation aux métiers de la musique. De nombreuses connaissances techniques nécessaires au travail musical s'acquièrent par les concerts, les tournées et les expérimentations tant techniques qu'artistiques avec différentes technologies et leurs possibilités. Un tel discours s'inscrit plus

\footnotetext{
5 Traduction libre de : " "experience" signifies a realm of rocky solidity and certainty, over against the airy abstraction of philosophy and social theory. It often confers authority when it is associated with the direct experience of life as opposed to "book learning", and it often serves as a common-sense, eyewitness guarantee of truth: "I know because I was there." "
} 
L'autoformation, nouveau paradigme de développement de carrière dans le contexte numérique? Regards sur le milieu musical montréalais

largement dans la valorisation du spectacle vivant dans les trajectoires de carrières musicales. Comme le soulignait Simon Frith (1983), la valeur d'une part importante des artistes - en particulier dans les genres associés au rock et ses déclinaisons est construite par et dans le discours de l'« authenticité » qui mobilise grandement le spectacle comme lieu d'établissement de la légitimité artistique. En d'autres mots, il s'agit de l'idée récurrente selon laquelle il faut faire ses classes pour prétendre au succès : " L'idéologie largement répandue parmi les fans, les musiciens.nes et les dirigeants de maisons de disques repose sur l'équation entre concert, authenticité musicale et le fait de payer de sa personne en tant qu'interprète ${ }^{6}$. » (Shuker, 2001, p. 107.) L'arrivée du rock et d'une génération plus jeune de musiciens.nes au cours des années 1960 et 1970 a stimulé le passage chez les techniciens de studios d'un statut d'artisan à celui d'artiste au sein de la production musicale, passant ainsi d'un monde de l'artisanat à un monde de l'art (Kealy, 1979). L'expérience de la scène permet non seulement d'acquérir des connaissances et de la crédibilité dans le milieu, mais aussi de partager et de comprendre les pratiques de création, l'utilisation de certains instruments, d'un certain son :

"C'est le genre de truc pour lequel ça prend des connaissances techniques qui ne sont pas nécessairement des trucs que t'apprends à l'école, de l'expérience pratique. Ça prend l'expérience, donc de l'expérience de scène. Les gens qui travaillent en prod [production] ont tous joué dans des bands généralement. Tous les gens que je connais qui font des grosses productions ont tous été dans le monde de la musique côté création d'une manière ou d'une autre. » (HR)

Outre ce qui relève de l'expérience de la scène, de nombreuses dimensions du métier et des activités se développent également au fil du quotidien, par l'essai-erreur. C'est notamment le cas de l'apprentissage des dimensions administratives des métiers de la musique :

« [Durant ma formation], j'aurais aimé ça qu'on me dise "OK faire de la musique ça implique être travailleur autonome,

\footnotetext{
${ }^{6}$ Traduction libre de : "The equation of live performance with musical authenticity and 'paying your dues' as a performer remains a widely held ideology among fans, musicians, and record company executives. "
} 


\section{Sylvain MARTET, Martin LUSSIER, Anouk BÉLANGER}

garde tes factures !" Moi j'ai appris ça moi-même, personne te dit comment être travailleur autonome, donc il y a des choses que, pour la majorité des gens, t'apprends au fur et à mesure. " (JT)

L'absence de ressources claires à mobiliser, par exemple de guides pratiques pour le développement de carrière, est un problème récurrent, qui doit cependant être mis en parallèle avec la grande diversité des parcours professionnels que l'on retrouve dans le milieu de la musique :

« J'ai appris au fil du temps, car ça fait quinze ans que je suis dans le business et que j'ai ma compagnie. [...] Je parle beaucoup à d'autres artistes aussi : "Toi, comment t'es organisé ?" C'est ça qui est dur, il n'y a pas vraiment de ressource pour aider. » (AZ)

L'expérience est mise de l'avant comme un mode autonome de formation dont le caractère autodidacte participe à la crédibilité de l'artiste dans son milieu. Cependant, même si la formation "sur le tas " est essentielle, elle se fait toujours au sein du milieu, à la fois avec et par les autres artistes, musiciens.nes et travailleurs.euses des industries musicales au sens large.

\section{Les communautés de pratiques et le partage des connaissances}

À partir de la fin des années 1990, l'offre de formation par les associations du milieu musical montréalais connaît une augmentation notable. Cela prend notamment forme dans l'organisation de formations par la SOPREF ou par l'ADISQ, comme mentionné plus haut, ainsi que par un nombre croissant d'associations professionnelles (éditeurs de musiques, auteurscompositeurs, musiciens.nes, etc.). Ces formations pratiques s'ajoutent à des journées d'étude, des conférences et des ateliers plus ponctuels, notamment en marge de festivals et d'événements musicaux qui rassemblent les acteurs de l'industrie. Malgré leur lien fort avec les industries musicales, ces formations demeurent peu valorisées par les personnes rencontrées. Cependant, le fait de se retrouver avec d'autres personnes du milieu musical dans ces formations semble apprécié. Le partage des connaissances au sein de la 
L'autoformation, nouveau paradigme de développement de carrière dans le contexte numérique? Regards sur le milieu musical montréalais

communauté est particulièrement valorisé :

"C'est souvent par les pairs la plupart des formations. Je sais qu'en marketing électronique, c'est une fille d'une autre maison de disque qui donnait la formation qui est très expérimentée là-dedans. [...] C'est beaucoup du mentorat par des gens qui ont de l'expérience là-dedans, qui font ça, c'est pas des professeurs, c'est des gens qui font ça et qui viennent donner leurs connaissances. " (DW)

La formation par le partage de l'expérience suppose la mise de l'avant d'espaces d'échange et de discussion qui ne se font pas du haut vers le bas, mais de pair à pair. Pour Lucy Green (2013), les modes informels d'éducation et de formation musicale seraient centraux pour les musiciens.nes populaires, même en contexte institutionnel (classes ou écoles de formation classiques). Pour elle, le partage informel que permettent la camaraderie ou les activités dans les interstices de l'institution forme un creuset idéal au développement des compétences et des attitudes nécessaires, comme l'évoque cette personne qui inclut dans les expériences de formation des rencontres annuelles sur l'état de l'industrie :

"C'est une présentation. II y a un PowerPoint et ils nous parlent des statistiques. On discute aussi, c'est des gens de l'industrie, c'est pas plein, on est une vingtaine. Chaque label parle un peu d'eux, c'est quoi leur réalité. » (ES)

Ce n'est pas uniquement pour le contenu de la rencontre - le cursus - qu'ES y participe, mais plutôt parce qu'elle sert d'espace d'échange de connaissances et de partage des expériences entre pairs. Si la dimension relationnelle et les opportunités de réseautage qu'entraîne cette offre de formation ponctuelle de l'industrie constituent des occasions fructueuses en soi, au-delà de l'acquisition de connaissance, le développement de contacts et de réseaux personnels est définitivement une valeur centrale :

" [Au Indie Week] il y a des ateliers, des conférences. C'est cool. Moi je capote ${ }^{7}$ pas sur les conférences, encore une fois, je suis relativement au courant de ce qui se passe, mais t'es là, tu parles avec des gens qui font la même affaire que toi. [...] Des fois c'est juste d'avoir des

${ }^{7}$ Capoter est un québécisme qui signifie s'enthousiasmer fortement. 


\section{Sylvain MARTET, Martin LUSSIER, Anouk BÉLANGER}

conversations allumées qui te font réfléchir sur les projets sur lesquels tu travailles, comment tu pourrais mieux les faire. » (FT)

II y a donc une double dimension dans les formations et les rencontres par l'industrie pour l'industrie: mobiliser des professionnels. les autour d'un contenu afin de rendre possible le partage de l'expérience entre pairs et permettre des échanges de pratiques. Ces moments de partage représentent des occasions de réseautage déterminantes pour le développement de la carrière des travailleurs.euses culturels: "dans la musique, les professionnels.les avancent dans leur carrière en connaissant les "bonnes personnes" et en ayant accès à un vaste réseau de contacts. Dans le même temps, les expérimentations esthétiques sont facilitées par de fréquents échanges face à face ${ }^{8}$ " (Cummins-Russell et Rantisi, 2012, p. 94). Cette importance est confirmée par de nombreuses personnes rencontrées dans le cadre de cette recherche :

"Tout ce qui est formation je vais toutes les prendre, je trouve ça intéressant pour les compétences mais aussi parce que tu rencontres des gens. Le réseautage c'est vraiment important. » (DW)

Ces formations ne sont cependant pas plébiscitées par tous. Une circulation informelle du savoir apparaît plus riche aux yeux de certains.es :

" Je retire bien plus à avoir une discussion d'une demiheure avec [une personne expérimentée] qui est super knowledgeable, j'en retire bien plus en une demi-heure de ça qu'en une formation. " (FT)

La discussion informelle constitue un véhicule majeur de circulation des connaissances, et ce dans divers contextes (festivals, spectacle, socialisation au quotidien, etc.). Les questions abordées peuvent être très techniques ou relever de tactiques plus globales d'adaptation à un contexte en mutation, tant par l'effet du numérique que du changement des modèles d'affaires qu'il induit.

8 Traduction libre de : « in music, professionals advance their careers by knowing the 'right people' and having access to a wide network of contacts, while aesthetic experimentation is facilitated by frequent face-to-face exchange. " 
L'autoformation, nouveau paradigme de développement de carrière dans le contexte numérique? Regards sur le milieu musical montréalais

Cette circulation informelle des savoirs au sein d'un réseau de pairs tend à se déployer à la fois à l'aune de la difficile définition des tâches et des métiers du milieu musical et du développement d'une communauté avec laquelle une forme d'attachement se dessine. Ainsi, HR met de l'avant la formation par les pairs dans son corps de métier, pour lequel il n'existe pas de formation initiale dédiée. L'introduction au métier et la formation se fait par l'expérience, accompagnée bénévolement d'un soutien technique et moral :

«Mon ami qui m'a engagé pour faire le job, il était tout le temps au téléphone avec moi les 2-3 premiers mois. Moi je suis pareil avec les gens sous mon aile, t'as pas le choix, parce que la personne que t'as engagée c'est ta responsabilité. » (HR)

La nécessité de former s'accompagne ici d'une responsabilité vis-à-vis de l'employeur, des clients et des partenaires. La crédibilité des travailleurs.euses est liée à leur capacité à accompagner les nouveaux venus dans leurs tâches, mais aussi à développer des réflexes de partage de connaissances. Chez tous nos répondants.es, c'est par les pairs que se développent les connaissances sur les métiers, le milieu et les pratiques :

« Vraiment, moi je pense même que c'est le principal, j'ai posé beaucoup de questions à des musiciens, des chanteurs rendus plus loin que moi pour être sûre, ou des gérants d'artistes en qui j'ai confiance. C'est important d'aller poser des questions. " (JT)

Ainsi, avec les nouveaux métiers et les tâches qui émergent du contexte des industries musicales en mutation, les réseaux de pairs représentent une ressource importante pour lutter contre l'incertitude. Comme l'indique BY, les ressources se trouvent beaucoup plus au sein de l'industrie musicale que sur les bancs d'école. L'enjeu étant de réussir à être connecté aux bons guides :

" Je pense aussi que quand tu rentres dans un milieu, il y a du monde qui sont plus au courant et qui finissent par te guider vers un endroit ou un autre, vers des ressources. » (BY).

Plus capital encore, la formation par les pairs et les partages sont identifiés comme une forme pratique de résistance à la crise 


\section{Sylvain MARTET, Martin LUSSIER, Anouk BÉLANGER}

des industries musicales et aux difficultés du milieu :

" Tout le monde veut que ça aille bien, que ça aille mieux. Tout le monde essaye de tâter le pouls, il y a une générosité, c'est quelque chose qui m'a agréablement surprise, je me suis jamais fait claquer la porte au nez par personne, tout le monde est très transparent, ouvert. Je pense que dans un moment où c'est tellement difficile d'avoir de l'argent, tous les artistes veulent s'assurer qu'ils ne se font pas [avoir], donc ça passe par le partage de l'information et de nos situations pour s'assurer que c'est fair pour tout le monde et qu'on encourage pas un [mauvais] système. » (JT)

Les personnes rencontrées dressent un portrait du milieu musical très ouvert au partage des connaissances. Les remarques telles que "Ouais on n'est pas en compétition, on s'aide. [...] II y a beaucoup de collégialité, tout le monde participe » (DW) sont récurrentes. Les professionnels.les de la musique se trouvent parfois dans des positions où ils cherchent des réponses à leurs questions, où ils sont demandeurs de ressources, curieux de pratiques, mais parfois aussi dans des positions de passeurs de savoir. Ainsi, AZ consacre une part de son temps à encourager le travail d'autres :

« J'essaie de travailler beaucoup avec des plus jeunes. [...] Je pense que ça c'est une clé aussi pour réussir. » (AZ)

Par conséquent, c'est d'abord par l'expérience et le partage de connaissances que l'adaptation et la formation aux changements circulent. Cependant, si le numérique impose des adaptations et des formations, il constitue également un espace de ressources vers lequel artistes et métiers de soutien se tournent.

\section{Le numérique comme ressource de compétences et de connaissances}

Une troisième voie de l'autoformation est directement liée à Internet, compris ici comme un espace permettant le développement de savoirs en autonomie. Le recours à Internet emprunte plusieurs voies, avec en premier lieu des formations en ligne (tutoriels, forums, conférences, etc.) répondant à des besoins ponctuels et spécifiques. Comme le souligne Paul 
L'autoformation, nouveau paradigme de développement de carrière dans le contexte numérique? Regards sur le milieu musical montréalais

Théberge (1997), ce type de ressources en ligne s'est développé à la fois en appui aux usagers de nouvelles technologies, comme lieu d'échange entre amateurs, et en renfort à la commercialisation de nouveaux produits. Pour nos répondants.es, ces ressources se caractérisent par leur diversité et la flexibilité qu'elles permettent. Les personnes rencontrées y accèdent par trois voies principales: sur conseil de leur entourage ; par le truchement des sites ou des plateformes de réseautage, notamment par des abonnements à des chaînes ou des groupes sur Facebook et YouTube; ainsi que par des portails numériques de regroupements plus ou moins formels et d'institutions ou de festivals. Parlant des ressources qu'elle utilise pour développer ses connaissances en marketing, et soulignant la flexibilité des savoirs en ligne, AZ nous dit :

" Je fais juste des formations en ligne. C'est vraiment juste... il faut que tu t'y intéresses. Je suis constamment en train de faire de la recherche, parce que ça change à chaque mois [...]. Si tu fais une formation et que tu laisses ça de même, dans deux ans ça ne servira plus à rien ce que tu as appris. » (AZ)

Les ressources en ligne s'intègrent mieux aux emplois du temps, puisque selon les projets dans lesquels ils sont impliqués, les travailleurs.euses de la musique n'ont pas toujours du temps pour des formations longues ou générales. Ainsi, FT profite en décalé des présentations de spécialistes lors de grands événements musicaux :

"South by Southwest mettent toutes les conférences sur un Soundcloud, alors moi quand je vais là, je vais pas m'asseoir dans les trucs pendant des heures à écouter du monde... » (FT)

Ces ressources s'intègrent également mieux au caractère ponctuel des questions, des défis et des problèmes rencontrés par les travailleurs. euses de la musique. Une question spécifique peut ainsi être résolue par le recours à des manuels en ligne, des vidéos ou encore des forums. Le développement de connaissances techniques sur les instruments électroniques, sur la production ou le mixage peut ainsi se faire en autoformation, indépendamment des écoles. HR indique ainsi avoir développé ses connaissances sur le matériel en " gossant sur des pitons [jouant avec des boutons]", en consultant Internet ou en 


\section{Sylvain MARTET, Martin LUSSIER, Anouk BÉLANGER}

discutant avec des techniciens, en ligne comme sur scène. Ce savoir-là est davantage valorisé dans le milieu, selon lui, qu'un diplôme qui ne garantit qu'une connaissance partielle du potentiel des outils :

« Dans mon cas, j'avais considéré les écoles comme des outils techniques, mais à la place de dépenser 13000 \$ sur un cours, j'ai décidé que j'allais juste acheter l'équipement et lire les informations sur Internet, parce que tous les tutoriaux, tous les manuels existent déjà. C'est une question d'avoir la patience et de vouloir apprendre par toimême. Tu peux te renseigner par toi-même, parce que le diplôme de l'école technique ne veut pas dire grand-chose dans le vrai monde. La personne qui sait comment utiliser des micros, des techniques d'enregistrement, qui a l'équipement chez lui, donc une connaissance intime des consoles, des tricks, cette personne-là va avoir plus de job qu'un étudiant je pense. " (HR)

Les ressources numériques sont particulièrement plébiscitées pour la veille sur les outils, mais aussi sur les nouveautés musicales, tant en création qu'en organisation. Lorsque FT écoute le nouveau morceau d'un artiste, il observe également la dimension stratégique qui l'accompagne :

« S'il y a un nouveau A $\$ A P$, je vois c'est quoi sa campagne, son roll out, je vois ce qu'ils font. Je le remarque, je le note dans ma tête. " (FT)

S'inspirer des pratiques des autres acteurs de l'industrie n'est pas nouveau en soi, cependant, au sein du contexte numérique, ce travail de veille est réalisé en partie par les artistes euxmêmes, du côté du développement d'affaires davantage que du côté de la création. C'est le cas d'AZ, qui cherche continuellement à améliorer sa compagnie :

« $\mathrm{A}$ tous les jours je suis en train de me tenir au courant dans des groupes Facebook. Je suis abonnée à un paquet de ressources, j'écoute des podcasts. Je pense que c'est vraiment la clé pour rester à flot. Ça prend une curiosité, une passion et un désir de réussir top niveau. » (AZ)

Ce travail de mise à jour et de veille constante, permis par les caractéristiques propres aux ressources en ligne, produit ainsi non seulement des connaissances à jour sur l'utilisation de 
L'autoformation, nouveau paradigme de développement de carrière dans le contexte numérique? Regards sur le milieu musical montréalais

certains outils, mais également sur les tendances esthétiques et de mise en marché en contexte numérique. Les personnes rencontrées ont également souligné le travail que constitue leur présence sur les réseaux socionumériques ainsi que la production d'images nécessaires à leur activité d'autopromotion :

« Aujourd'hui je pense que l'image est importante, peut-être pas pour tous les artistes, mais pour beaucoup. Le fait de s'afficher et d'être présent sur Internet, que ce soit sur un cellulaire ou une caméra, c'est nécessaire et le travail existe, c'est là. » (NZ)

Plusieurs recherches (Baym, 2018 ; Creton, 2018) soulignent l'importance que ces tâches occupent dans les carrières musicales. JT partage cette conviction, ajoutant que « c'est un business de gérer des réseaux sociaux, travailler l'image que t'as par rapport à tes fans ". Devant une telle tâche, les ressources principales pour s'inspirer et pour se former se retrouvent en partie auprès des pairs, et surtout en ligne :

« la formation je la fais moi-même en la trouvant sur Internet et en discutant, mais oui je m'informe énormément. Une formation en réseaux sociaux, c'est juste en observant, c'est de la recherche au final, même si c'est regarder des comptes Instagram, j'apprends de ça. » (JT)

Ces activités n'ont pas été apprises sur les bancs de l'école pour les personnes rencontrées. Si une musicienne nous a indiqué avoir suivi des formations professionnelles pour gérer ses réseaux sociaux, les autres se fient plutôt aux ressources qu'elles peuvent trouver en ligne. Dans tous les cas, il s'agit de parcours combinant la mobilisation de diverses formes de connaissances au gré des besoins rencontrés ou des projets en cours.

La provenance et l'adéquation des ressources en ligne aux outils et aux pratiques du milieu sont un enjeu important. En ce sens, une limite est identifiée par les personnes rencontrées : celle de l'orientation dans les savoirs en ligne. Parlant spécifiquement des stratégies de diffusion sur Internet, CX souligne que les meilleures ressources sur ce sujet se trouvent en ligne... mais que toutes ne sont pas pertinentes :

« La grande qualité de l'Internet c'est que l'Internet est la formation en soi. Dès que tu comprends Google, tu peux 


\section{Sylvain MARTET, Martin LUSSIER, Anouk BÉLANGER}

apprendre [comment fonctionne] Internet en allant sur Internet. C'est comme du cannibalisme ! [...] Le problème avec l'Internet c'est qu'il y a beaucoup de savoirs qui sont erronés, mais il y a beaucoup de savoirs, alors tu peux le filtrer et trouver quelque chose qui est relativement efficace. » (CX)

Les ressources disponibles en ligne ont des provenances diverses, tant amateurs que professionnelles, ou même commerciales. On comprend ici que l'enjeu majeur devient alors celui de l'orientation en ligne vers les bonnes ressources. Or ces capacités sont le produit de leur expérience, selon nos répondants.es. L'autoformation assistée par Internet suppose donc aussi des connaissances et des savoirs de base ainsi qu'une capacité à la recherche autonome :

" N'importe quelle opération en ligne, si je ne sais pas comment la faire, je n'ai pas besoin d'aller faire une formation. Je suis rendu capable d'aller trouver mes réponses. Mais ça m'a pris du temps. T'as besoin d'une connaissance de base avant de développer des systèmes pour t'auto-éduquer. » (AZ)

Les savoirs implicites, propres aux modes informels d'éducation, participent ainsi également des modes de formation mobilisant les ressources numériques. Une part des savoirs du milieu musical dépend ainsi d'un ensemble de non-dits qui permettent une utilisation adéquate des ressources numériques.

\section{Conclusion}

Dans le contexte numérique, la croissance de l'industrie de la formation en musique ainsi que le rôle des écoles et des institutions de formations postsecondaires dans la professionnalisation du milieu musical laissent présager leur attractivité pour les travailleurs.euses du milieu musical. Or si la moitié de nos répondants.es ont suivi une formation musicale postsecondaire et formelle, un seul la met clairement de l'avant pour expliquer le développement de sa carrière.

Pour Porcello (2004) ainsi que pour Bellavance et Laplante (2002), les formations " en classe " sont de bonnes façons d'acquérir rapidement des savoirs implicites propres au milieu musical, tout comme un langage commun. Pourtant, les 
L'autoformation, nouveau paradigme de développement de carrière dans le contexte numérique? Regards sur le milieu musical montréalais

personnes rencontrées dans le cadre de cette recherche soulignent que de nombreux savoirs sont impossibles à acquérir de cette façon et qu'ils proviennent plutôt d'une formation « en autodidacte " ou en continu. Une mise en doute de la capacité des formations professionnalisantes de répondre à la vélocité des changements et des besoins afférents du milieu est largement partagée. Pour les participants.es, ce doute est principalement fondé sur trois arguments: l'impossibilité ressentie d'enseigner les tâches concrètes de leur métier, la préférence pour des échanges au sein de communautés de pairs et la rapidité avec laquelle les outils numériques en création, en gestion ou en réseautage social changent, voire deviennent obsolètes. Les institutions classiques d'enseignement, par exemple les conservatoires, ne semblent pas disposer de moyens pour adapter leurs programmes au rythme de transformation de la réalité du travail en musique dans le contexte numérique. Ce clivage dans les temporalités participe au choix des personnes rencontrées de chercher des ressources hors des réseaux scolaires de formation.

Dans un contexte où les travailleurs.euses du milieu musical ont tendance à occuper plusieurs fonctions sans avoir nécessairement de formation dans chacune des dimensions de leur métier, les recours aux communautés de pratiques, aux ressources en ligne, à l'expérimentation et à l'autoformation sont légion, tant pour s'adapter au contexte numérique qu'aux nouveaux modèles et rapports d'affaires. Notre projet montre très clairement que les informations et les savoirs circulent dans le milieu sous des modalités variées. Cette circulation des connaissances est une tactique de résistance à la précarisation du milieu, mais aussi une tactique d'adaptation à ces changements. La notion d'expérience qui se forge en partie de manière autonome en ligne se couple avec la formation par les pairs, qu'elle soit institutionnalisée par des associations ou informelle, liée à une situation particulière ou à un travail presque imperceptible de veille et de formation continue.

La « rencontre » entre l'entrepreneuriat créatif et l'omniprésence d'outils numériques, en création, en diffusion et dans l'organisation du travail au quotidien dessine un nouveau rapport à la formation. Celle-ci n'est plus envisagée comme une étape de préparation au travail, mais plutôt comme une partie continue du travail, ou plutôt comme plusieurs parties du travail, 


\section{Sylvain MARTET, Martin LUSSIER, Anouk BÉLANGER}

séparées selon des domaines précis : formation technique aux outils de création, formation au travail en équipe, formation en administration, formation en gestion des réseaux sociaux... Les parcours de formation qui accompagnent les parcours artistiques sont variés et intègrent autant des formes institutionnelles que des réseaux informels. L'école est ainsi mise à profit, au même titre que d'autres sources, telles qu'un forum spécialisé, une journée de perfectionnement organisée par une association professionnelle ou encore l'expérimentation et l'expérience dans des parcours en évolution constante. La formation est de moins en moins attachée à l'école et à un moment fondateur dans les parcours. Accompagnée par le numérique, entre autres, elle est désormais éclatée, individualisée et continue.

\section{Références}

Bacache-Beauvallet, M., Bourreau, M. et Moreau F. (2011). Portrait des musiciens à l'heure du numérique. Paris, France : Éditions Rue d'Ulm.

Baker, S. et Hesmondhalgh, D. (2011). Creative Labour: Media Work in Three Cultural Industries. New York, NY : Routledge.

Barcelo, Y. (1990, 5 mai). Musitechnic, modèle unique d'école de musique dans la francophonie. Les Affaires, 16.

Bastard, I., Bourreau, M., Maillard, S. et Moreau F. (2012). De la visibilité à l'attention: les musiciens sur Internet. Réseaux, (175), 19-42.

Baym, N. K. (2018). Playing to the Crowd: Musicians, Audiences, and the Intimate Work of Connection. New York, NY: New York University Press.

Bellavance, G. et Laplante, B. (2002). Professionnalisation et socialisation du champ artistique : la formation professionnelle des artistes du XXème siècle. Dans D. Lemieux et al. (dir.), Traité de la culture (p. 315-339). Sainte-Foy, Canada: Les éditions de l'IQRC.

Bérubé, M. (2005). Experience. Dans T. Bennett, L. Grossberg, et M. Morris (dir.), New Keywords: A Revised Vocabulary of Culture and Society (p. 121-123). Oxford, Royaume-Uni : Blackwell. 
L'autoformation, nouveau paradigme de développement de carrière dans le contexte numérique? Regards sur le milieu musical montréalais

Beuscart, J.-S. (2008). Sociabilité en ligne, notoriété virtuelle et carrière artistique. Les usages de MySpace par les musiciens autoproduits. Réseaux, (152), 139-168.

Bourgault-Côté, G. (2009, 21-22 mars). Les gros blues du disque. Le Devoir, A12.

Brunet, A. (2015, 3 décembre). Le Studio Victor ferme ses portes. La Presse, A20.

Bureau, M.-C. (2006). L'entrepreneuriat culturel et associatif : une question d'économie politique. Économie et solidarités, 36(1), 126-140.

Bureau, M.-C. et Shapiro, R. (2009). Introduction. « Et à part ça, vous faites quoi ? ». Dans M.-C. Bureau, M. Perrenoud et R. Shapiro (dir.), L'artiste pluriel. Démultiplier l'activité pour vivre de son art (p.17-31). Villeneuve d'Ascq, France: Presses universitaires du Septentrion.

Charette, M. (2000, 12 février). Son et informatique : un heureux mariage. La Presse, 18.

Chircu, A. M. et Kaufmann, R. J. (1999). Strategies for internet middleman in the intermediation/disintermediation/ reintermediation cycle. Electronic Markets: The International Journal of Electronic Commerce and Business Media, 9(2), 109-117.

Creton, C. (2018). To pay or not to pay : les musiciens à notoriété locale face à la publicité ciblée sur Facebook. Les enjeux de l'information et de la communication, 19(2), 15-28.

CRHSC. (2011). Culture 3.0. Impact des technologies numériques émergentes sur les ressources humaines du secteur culturel. Ottawa, Canada : Conseil des ressources humaines du secteur culturel (CRHSC).

Culture Montréal. (2016). Adapter notre écosystème culturel à l'ère du numérique. Enjeux prioritaires. Montréal, Canada: Culture Montréal.

Cummins-Russell, T. A. et Rantisi, N. M. (2012). Networks and place in Montreal's independent music industry. The Canadian geographer, 56(1), 80-97.

Daoust-Boisvert, A. (2011, 13-14 août). Les arts à l'école : si on jouait. Le Devoir, F6. 


\section{Sylvain MARTET, Martin LUSSIER, Anouk BÉLANGER}

Frith, S. (1983). Sound Effects: Youth, Leisure, and the Politics of Rock. Londres, Royaume-Uni : Constable.

Green, L. (2013). Music, Informal Learning and the School: A New Classroom Pedagogy. Farnham, Rpyaume-Uni : Ashgate Publishing.

Greffe, X. (2012). L'artiste-entreprise. Paris, France : Dalloz.

Grenier, L. (2011). "Crise » dans les industries de la musique au Québec: ébauche d'un diagnostic. Recherches sociographiques, 52(1), 27-48.

Hracs, B. J., Seman, M. et Virani, T. E. (dir.). (2016). The Production and Consumption of Music in the Digital Age. New York, NY/Londres, Royaume-Uni : Routledge.

Kealy, E. R. (1979). From Craft to Art: The Case of Sound Mixers and Popular Music. Dans S. Frith et A. Goodwin (dir.), On Record: rock, pop, and the written word (p. 172-184). New York, NY/ Londres, Royaume-Uni : Routledge.

Le Guern, P. (2012). Irréversible ? Musique et technologies en régime numérique. Réseaux, (172), 29-64.

Lussier, M. (2017). Éducation, jeunes et musiques émergentes à Montréal : travail pédagogique et production de la relève d'une culture jeune. Dans S. Octobre et C. Dallaire (dir.). Jeunes et cultures: dialogue franco-québécois (p. 167-184). Québec, Canada : Presses de l'Université Laval.

McRobbie, A. (2002). Clubs to Companies: Notes on the Decline of Political Culture in speeded up creative worlds. Cultural Studies, 16(4), 516-531.

Menger, P.-M. (2002). Portrait de l'artiste en travailleur. Paris, France : Le seuil.

Morris, J. W. (2014). Artists as entrepreneurs, fans as workers. Popular Music and Society, 37(3), 273-290.

Papineau, P. (2017, 14 février). Numérique et arts de la scène : l'urgence côtoie l'inquiétude. Le Devoir, B8.

Porcello, T. (2004). Speaking of Sound: Language and the Professionalization of Sound-Recording Engineers. Social Studies of Science, 34(5), 733-758. 
L'autoformation, nouveau paradigme de développement de carrière dans le contexte numérique? Regards sur le milieu musical montréalais

Prior, N. (2012). Musiques populaires en régime numérique. Acteurs, équipements, styles et pratiques. Réseaux, (172), 66-90.

Québec (2014). Pour occuper l'espace numérique. Stratégie culturelle numérique du Québec. Québec, Canada : Ministère de la Culture et des Communications.

Shuker, R. (2001). Understanding Popular Music (2 ${ }^{\text {ème }}$ édition). Londres, Royaume-Uni/New York, NY : Routledge.

Sinigaglia-Amadio, S. et Sinigaglia, J. (2017). Temporalités du travail artistique: le cas des musicien.ne.s et des plasticien.ne.s. Paris, France : La documentation française.

Théberge, P. (1997). Any Sound You Can Imagine: Making Music/Consuming Technology. Hanover, PA/Londres, Royaume-Uni : Wesleyan University Press.

Watson, A. (2016). Laptops, Pro Tools, and File Transfer Protocols: On the Intensification and Extensification of Recording Work in the Digital Age. Dans B. J. Hracs, M. Seman et T. E. Virani (dir.). The Production and Consumption of Music in the Digital Age (p. 20-33). New York, NY/Londres, Royaume-Uni : Routledge. 